\title{
Searches for new heavy resonances and extra dimensions at CMS
}

\author{
Francesco Romeo* \\ IHEP of Beijing, \\ On behalf of the CMS collaboration \\ E-mail: francesco.romeodcern.ch
}

Final states with a pair of particles are sensitive to many models beyond the standard model (SM) and allow for a clean search for both new narrow resonances and broad deviations from SM predictions. The most recent results from searches with dileptons, leptons plus missing transverse energy, and dijets are presented using $19.7 \mathrm{fb}^{-1}$ of pp collision data collected by the CMS detector with $\sqrt{s}=8 \mathrm{TeV}$ at the LHC. No evidence is observed for physics beyond the SM and 95\% confidence level (CL) limits are set on parameters from a number of theoretical models such as $Z^{\prime}, W^{\prime}$, RS gravitons, large extra dimensions scenarios, and contact interactions.

The XXIII International Workshop on Deep Inelastic Scattering and Related Subjects April 27 - May 1, 2015

Southern Methodist University

Dallas, Texas 75275

\footnotetext{
* Speaker.
} 


\section{Introduction}

The standard model of particles physics (SM) describes the properties of all known matter constituents and the forces between them. Despite its successes, there are solid indications that it is not a complete theory and is today generally accepted rather as a low-energy remnant, or a visible part, of a more fundamental theory. In attempts to extend the SM, several beyond the SM scenarios have been proposed that foresee the existence of new heavy resonances and extra dimensions. Some of the most popular theories of interest for this proceeding are briefly introduced below.

Sequential Standard Model (SSM) [1]. It is a benchmark model used in searches of new heavy spin 1 bosons denoted as $Z^{\prime}$, if neutral, or $W^{\prime}$, if charged, that have the same couplings to quarks and leptons as the $\mathrm{SM} Z$ and $W$ bosons.

Grand Unified Theories (GUT) [2]. In this model the strong and the electroweak interactions are merged into a single interaction, described by a higher symmetry group. In the GUT-inspired $E_{6}$ scenario the symmetry is broken down by the following pattern:

$$
E_{6} \rightarrow S O(10) \times U(1)_{\psi} \rightarrow S U(5) \times U(1)_{\chi} \times U(1)_{\psi} \rightarrow S U(3)_{C} \times S U(2)_{L} \times U(1)_{Y} \times U(1)_{\theta_{E_{6}}},
$$
where $U(1)_{\theta_{E_{6}}}$ is believed to be broken at the TeV scale. Only one linear combination remains light: $Z^{\prime}=Z_{\chi}^{\prime} \cos _{\theta_{E_{6}}}+Z_{\psi}^{\prime} \sin _{\theta_{E_{6}}}, \theta_{E_{6}}$ being a free parameter of the model in the range of $-90^{\circ} \leq \theta_{E_{6}} \leq 90^{\circ}$.

Supersymmetry (SUSY) [3]. It offers a solution to the hierarchy problem and provides a candidate for dark matter. The most general renormalisable superpotential in SUSY introduces interactions which violate both baryon and lepton number. In many models of SUSY, these terms are removed from the classical Lagrangian by requiring an additional $\mathbb{Z}_{2}$ symmetry, called R-parity. Instead, the baryon triality symmetry $\left(B_{3}\right)$ is considered in the following. It cancels baryon number violating terms and stabilises the proton, leading to the presence of trilinear R-parity violating (RPV) couplings $\lambda_{i j k}^{\prime}$ and $\lambda_{i j k}$ of the superpotential. The $B_{3}$ symmetry allows for a resonant production and decay of a $\tilde{v}_{\tau}$ through the process $d \bar{d}\left(\lambda_{311}^{\prime}\right) \rightarrow \tilde{v}_{\tau} \rightarrow e \mu\left(\lambda_{132}\right)$.

Models with extra dimensions are usually introduced to find an answer to hierarchy problem.

Arkani-Hamed, Dimopolous, and Dvali (ADD) [4]. In this model the ordinary Planck scale $\left(M_{P l}\right)$ is related to the fundamental Planck scale $\left(M_{D}\right)$ via the formula: $M_{P l}^{2}=M_{D}^{2+n} \times R^{n}$, where $n$ is the number of large extra dimensions and $R$ their size. The parameter $\eta_{G}$ regulates the cross section in presence of ADD phenomena and it is set as $\eta_{G}=1 / \Lambda_{T}^{4}=\mathscr{F} / M_{S}^{4}$, where $M_{S}$ is the ultraviolet cutoff of the sum over Kaluza-Klein excitations in virtual gravition exchange $\left(M_{S} \sim M_{D}\right.$ in $4+n$ dimensional space) and $\mathscr{F}$ is a parameter that may be equal to 1 in the GRW (Giudice, Rattazzi, Wells) convention or $\frac{2}{n-2}$, for $n>2$, in the HLZ (Han, Lykken, Zhang) variant.

Universal Extra Dimension (UED) [5]. This model is based on an extended space-time with one additional compact dimension of radius R. It foresees a tower of $W_{k k}^{n}$ Kaluza-Klein excitations, of which LHC is expected to be sensitive only to $n=2 . W_{k k}^{n}$ mass depends on the radius R (larger radius corresponds to smaller masses), while the production cross section times the branching fraction to standard model fermions on the parameter $\mu$ (it goes to zero as $\mu$ goes to zero).

Randall-Sundrum (RS) [6]. This model foresees one additional dimension with a strong curvature. Kaluza-Klein gravitons can couple to fermions through coupling parameter $k / \bar{M}_{P l}$, where $k$ is the unknown curvature scale of the extra dimension and $\bar{M}_{P l}$ is the reduced Planck scale.

Contact Interaction (CI) [7]. Contact interaction is a feature of a compositeness scenario in which quarks and leptons are assumed to have an internal substructure that should become manifest at some sufficiently high energy scale, the compositeness scale $\Lambda$. 

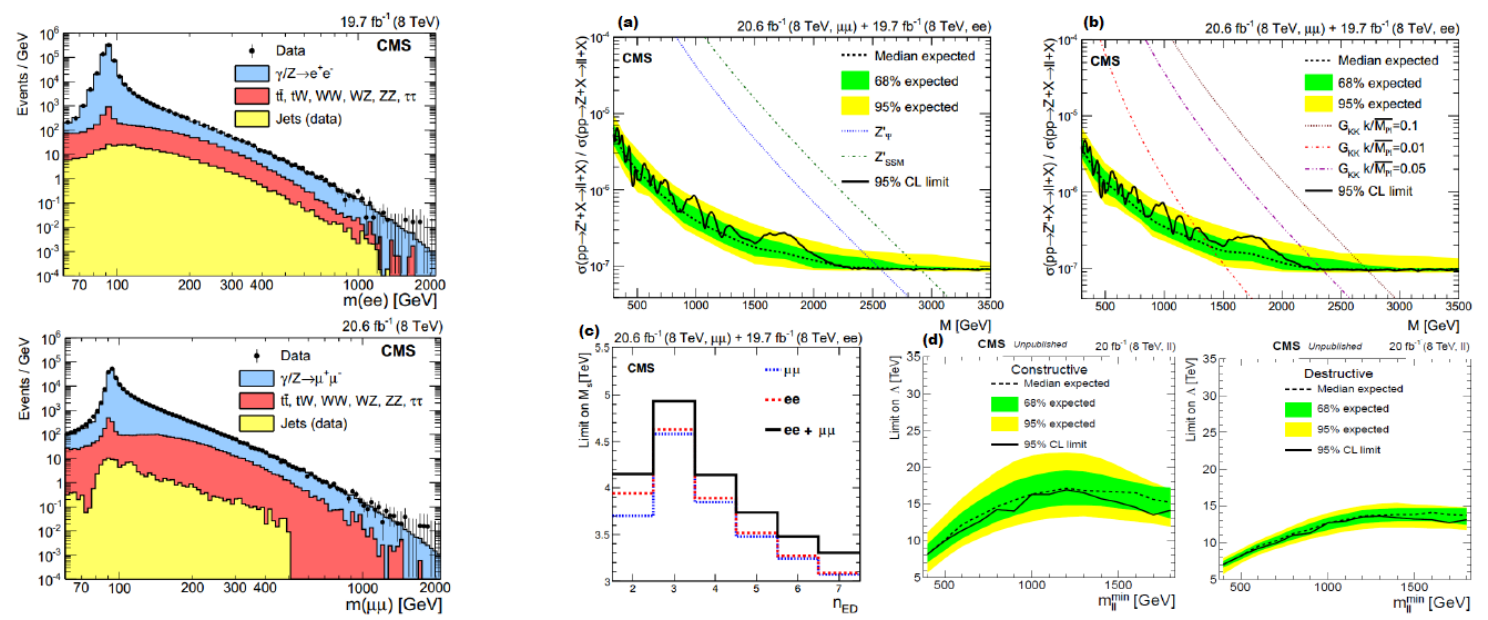

Figure 1: Left: The $e e$ (up) and $\mu \mu$ (down) invariant mass spectrum for data (points) and SM expectations (histograms). Right: The 95\% CL exclusion bounds for (a) $Z_{S S M}^{\prime}$ and $Z_{\psi}^{\prime}$, (b) RS, (c) ADD, and (d) CI models described in Section 1.

In this proceeding the most recent results of searches for new heavy resonances and extra dimensions are presented using the $19.7 \mathrm{fb}^{-1}$ of pp collision data collected by the CMS detector [8] with $\sqrt{s}=8 \mathrm{TeV}$ at the LHC. Signatures with a pair of particles and particles plus missing transverse energy $\left(Z_{T}\right)$ are considered for different final states including: $e e$ and $\mu \mu, e \mu, e \mu+Z_{T}, e$ or $\mu$ or $\tau_{h}+B_{T}$ (where $\tau_{h}$ represents a hadronically decaying tau), and $j j$. A complete description of the objects used in the analyses described below as well as of the systematic uncertainties treatment can be found in the corresponding references.

\section{The $e e$ and $\mu \mu$ final states}

In this section an investigation for evidence of physics beyond the SM using the dilepton (dielectron and dimuon) invariant mass spectra is described. A complete description is given in [9].

Two isolated, same-flavour leptons are required. Electrons must have transverse energy $E_{T}>$ $35 \mathrm{GeV}$ and absolute value of the pseudorapidity $|\eta|<2.5$. Muons must have transverse momentum $p_{T}>45 \mathrm{GeV}$ and $|\eta|<2.1$ (2.5) for leading (sub-leading) candidate. Muon pairs are also required to have opposite charges and that their opening angle be less than $\pi-0.02$ radians.

The principal SM background that contributes to the dilepton invariant mass spectra is the Drell-Yan production that is estimated from simulation. The contamination due to prompt lepton pairs from $t \bar{t}, t W$, and diboson production is also evaluated using simulation, after validation with respect to data. Moreover jets, from multijet and vector boson plus jets final states, may pass the electron selection. This misidentification probability is measured from data and is used to weight events that do not pass electron selection to provide an estimation of this reducible background.

The dilepton invariant mass spectra are shown in Fig. 1 (left), where the data are compared with the expected SM backgrounds. The simulated background contribution is normalised to the number of events in the data in the region of $60<m_{\ell \ell}<120 \mathrm{GeV}$.

No evidence for beyond SM physics is observed and 95\% confidence level limits are set on parameters from a number of new physics models. In the case of new narrow resonance searches, the ratio of the cross sections for producing dilepton final states, $R_{\sigma}=\frac{\sigma\left(p p \rightarrow Z^{\prime}+X \rightarrow \ell \ell+X\right)}{p p \rightarrow Z+X \rightarrow \ell+X}$, is used to interpret observed events. The cross sections correspond to a mass range of $\pm 5 \% \sqrt{s}$ about the $Z^{\prime}$ on-shell mass and a mass range of $\pm 30 \mathrm{GeV}$ about the $Z$ peak. This $Z^{\prime}$ mass window is designed to allow for straightforward reinterpretation of the limits derived in this study in the context of models 

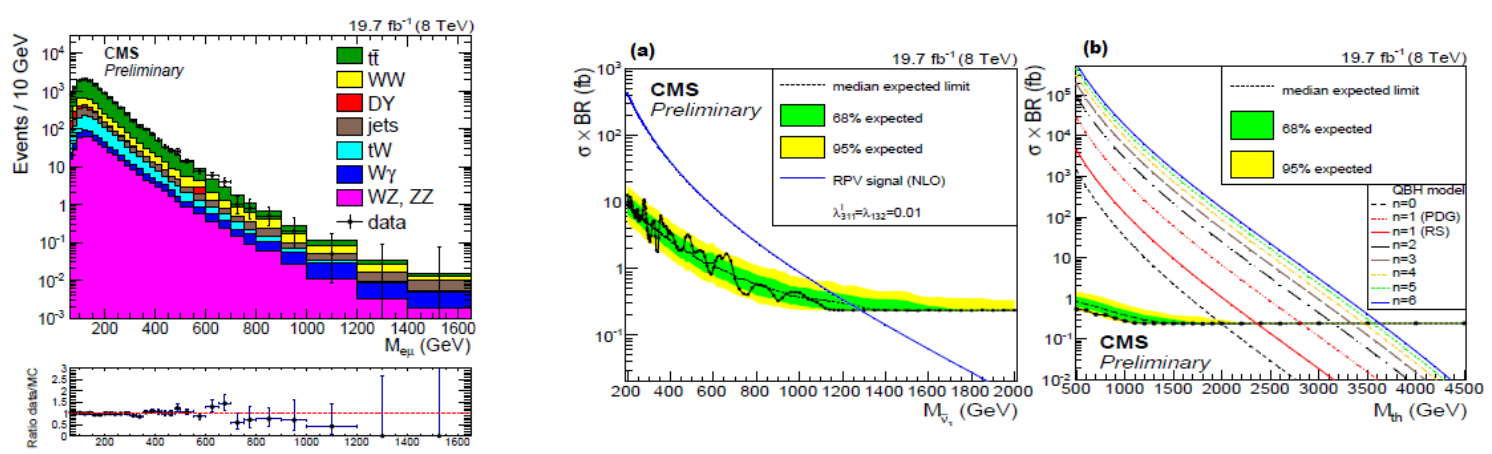

Figure 2: Left: The $e \mu$ invariant mass spectrum for data (points) and SM expectations (histograms). Right: The 95\% CL exclusion regions considering (a) RPV SUSY and (b) QBH models described in Section 1 and Section 3.

not specifically addressed. In this analysis a $Z_{S S M}^{\prime}$ lighter than $2.90 \mathrm{TeV}$, a $Z_{\psi}^{\prime}$ lighter than $2.57 \mathrm{TeV}$, and RS Kaluza-Klein gravitons with masses below 2.73, 2.35, and $1.27 \mathrm{TeV}$ for couplings $k / \bar{M}_{P l}$ of $0.10,0.05$, and 0.01 respectively are excluded. Lower limits are established also for the parameters of two models from which non-resonant mass shapes are expected: $M_{S}$ of the ADD model from 4.90 to $3.30 \mathrm{TeV}$, with $n$ varying from 3 to 7 ; and $\Lambda$ of the $\mathrm{CI}$ framework for values of $13.10 \mathrm{TeV}$ and $16.90 \mathrm{TeV}$ for destructive and constructive interference. Figure 1 (right) shows the exclusion plots for the aforementioned interpretations.

\section{The $e \mu$ final state}

In this section a measurement looking for lepton flavour violating processes in the $e \mu$ final state is reported. A detailed description is provided in [10].

The search is designed by requiring a pair of a prompt isolated electron and muon with $E_{T}>$ $35 \mathrm{GeV}(|\eta|<2.5)$ and $p_{T}>45 \mathrm{GeV}(|\eta|<2.1)$.

The analysis considers two main sources of background: one is given by two leptons originating from Drell-Yan, $t \bar{t}, t W$, and diboson processes, which are estimated from simulation; the other is due to a misidentified electron from a jet that is paired to a muon, e.g. as in $W+$ jet process, and is estimated using the same method mentioned in Section 2.

The $e \mu$ invariant mass distribution is shown in Fig. 2 (left) for the data and the SM expectations. No significant excess of the data with respect to the SM expectation is found in the measured invariant mass distribution and the 95\% CL limit on the signal cross section times branching ratio for the RPV SUSY $\tilde{v}_{\tau}$ in the $e \mu$ final state is set. For couplings $\lambda_{311}^{\prime}=\lambda_{132}=0.01$ a mass below $1.28 \mathrm{TeV}$ is excluded. Limits are also set in the $M_{\tilde{v}_{\tau}}-\lambda_{311}^{\prime}$ parameter plane as a function of a fixed value of $\lambda_{132}$ as reported in [10]. The results are further interpreted considering the production of spin-0, colourless, neutral non-thermal quantum black holes (QBH) [11] in a model with lepton flavour violation, in which the production cross section depends on the threshold mass $M_{t h}$ for producing QBH and the number of extra dimensions $n$. The 95\% CL exclusion limits for these models are shown in Fig. 2 (right).

\section{The $e \mu+Z_{T}$ final state}

In this section a study looking for new physics beyond the SM in the high-mass ditau final state with one tau decaying in the electron channel and one tau decaying in the muon channel is presented. A complete description is reported in [12].

The selection of signal events is performed requiring muon $p_{T}>20 \mathrm{GeV}(|\eta|<2.1)$, electron $E_{T}>20 \mathrm{GeV}(|\eta|<2.5)$, and $E_{T}>20 \mathrm{GeV}$. Electrons and muons are paired if they have oppo- 
site charges and satisfy the condition $\cos \Delta \phi(e, \mu)<-0.95$, where $\Delta \phi(e, \mu)$ is the difference in the azimuthal angle among the selected candidates. Events are further sought to have no b-tagged jet.

The $t \bar{t}$ background is estimated from data by selecting events of the signal region that have 2 b-tagged jets and weighting these events by the 0 -jet to 2 -jet ratio, measured from data in an independent control region. The multijet contribution is estimated considering like-sign $e \mu$ candidates and subtracting Monte-Carlo (MC) expectation of non-multijet backgrounds from data. The other relevant backgrounds are Drell-Yan and $W W$ that are estimated from simulation, after validation with respect to data.

The invariant mass of e, $\mu$, and $\mathbb{E}_{T}$ is shown in Fig. 3 (left). A good agreement is found between the observed data and the expected SM background. $Z_{S S M}^{\prime}$ with masses less than 1.30 $\mathrm{TeV}$ and $Z_{\psi}^{\prime}$ with masses less than $0.81 \mathrm{TeV}$ at $95 \% \mathrm{CL}$ are excluded. $\Lambda_{T}$ parameter in the GRW convention of the ADD model is excluded up to $2.80 \mathrm{TeV}$ at $95 \% \mathrm{CL}$. This value corresponds to 95\% CL upper limits of $3.33 \mathrm{TeV}$ and $2.23 \mathrm{TeV}$ (for $n=3$ and 7) on the parameter $M_{s}$ in the HLZ variant of the ADD model. The exclusion limits for these models are shown in Fig. 3 (right).
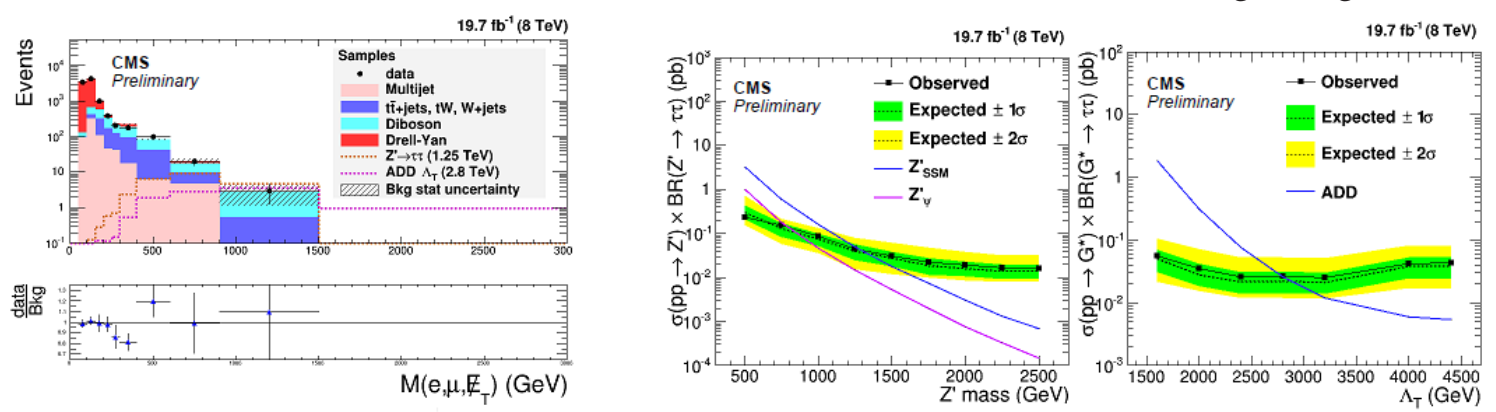

Figure 3: Left: The $e, \mu$, and $\mathbb{E}_{T}$ invariant mass spectrum for data (points) and SM expectations (histograms). Right: The 95\% CL exclusion limits for (a) $Z_{S S M}^{\prime}$ and $Z_{\psi}^{\prime}$, and (b) ADD models described in Section 1.

\section{The $\ell+\not E_{T}$ final states}

In this section a measurement aiming to look for new phenomena beyond the SM in the final state with a lepton plus $\not_{T}$, where the lepton can be an electron, a muon, or a hadronically decaying tau is described. A full description is given in [13] and [14].

$E_{T}>100 \mathrm{GeV}(|\eta|<2.5), p_{T}>45 \mathrm{GeV}(|\eta|<2.1)$, and $p_{T}>100 \mathrm{GeV}(|\eta|<2.3)$ is sought for e, $\mu$, and $\tau_{h}$ respectively. Since the lepton $p_{T}$ and the $\mathbb{E}_{T}$ are expected to be nearly back-to-back in the transverse plane and balanced in transverse energy, $0.4(0.7)<p T^{\ell} / E_{T}<1.5$ and $\Delta \phi\left(\ell, \mathbb{E}_{T}\right)>2.5(2.4)$ is also demanded for the final state with e, $\mu\left(\tau_{h}\right)$.

The primary source of contamination is due SM $W \rightarrow \ell v$ decay and is estimated from MC simulation. The contribution due to jets from multijets events misidentified as e and $\tau_{h}$ is estimated from data: the shape is obtained in a region with a non-isolated lepton; the normalisation is determined in a signal-free reference region with $p T^{\ell} / B_{T}>1.5$.

The transverse mass of the leptons and $E_{T}, M_{T}\left(\ell, \not_{T}\right)=\sqrt{2 \cdot p_{T}^{l} \not_{T}(1-\cos \Delta \phi)}$, is shown in Fig. 4 (left). These spectra show no significant excess of data compared to the SM expectations. A $W_{S S M}^{\prime}$ boson, that does not interfere with the SM $W$ boson, has been excluded at 95\% CL for masses up to $3.28 \mathrm{TeV}$, combining the electron and muon channel, and $2.7 \mathrm{TeV}$ considering the $\tau_{h}$ channel. The expected impact of $W-W^{\prime}$ interference on the shape of the $W$ boson, the $M_{T}\left(\ell, E_{T}\right)$ distribution, is fully taken into account in the analyses dealing with e and $\mu$ (see [13]). 

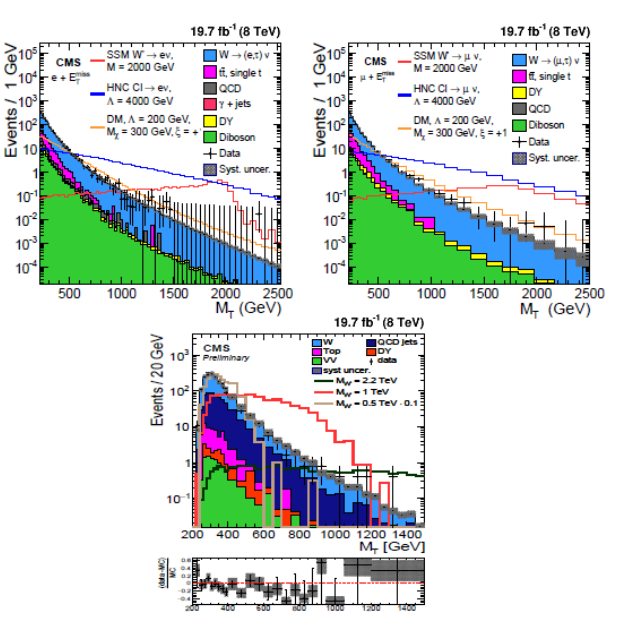
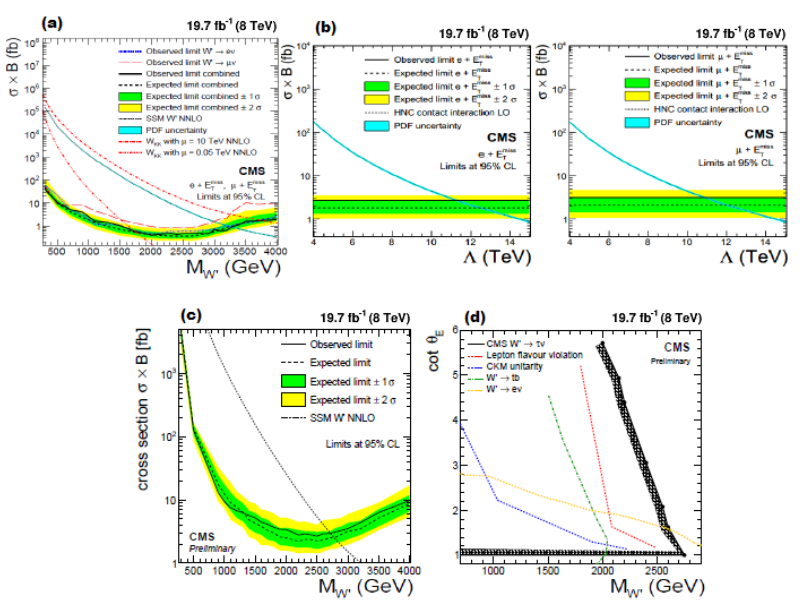

Figure 4: Left: The $\ell$ plus $Z_{T}$ transverse mass spectrum for e, $\mu$ (up) and $\tau_{h}$ (down) for data (points) and SM expectations (histograms). Right: The 95\% CL exclusion bounds considering (a) $W_{S S M}^{\prime}$ and UED, (b) CI models (see Section 1) in searches with e, $\mu$, and (c) $W_{S S M}^{\prime}$, (d) NUGIM models in searches with $\tau_{h}$ (see Section 5).

The e, $\mu$ channels interpret the results considering also the UED model, for which $W_{K K}^{2}$ is excluded for masses below $1.74 \mathrm{TeV}$, being $\mu=0.05 \mathrm{TeV}$, and for masses below $3.71 \mathrm{TeV}$, being $\mu=10 \mathrm{TeV}$, and a CI model where the helicity is not conserved, for which $\Lambda$ of $11.30 \mathrm{TeV}$ in the electron channel, $10.90 \mathrm{TeV}$ in the muon channel, and $12.40 \mathrm{TeV}$ from the combination of both are excluded. The $\tau_{h}$ channel further interprets the results in terms of a non-universal gauge interaction model (NUGIM) [15] in which the SM SU(2) group is mixed with an extended SU(2) group through the angle $\theta_{E}$ which modifies the couplings and for $\cot \theta_{E}$ favours third-generation of fermions. This search sets the best limit up to date on $\cot \theta_{E}$ (see [14]). Limit plots are reported in Fig. 4 (right).

\section{The $j j$ final states}

In this section a search for new heavy states and QBH from a dijet signature is summarised. The measurement is presented in more detail in [16].

All the jets in the analysis are required to have $p_{T}>30 \mathrm{GeV}$ and $|\eta|<2.5$. The dijet system is then composed by the two jets with largest $p_{T}$ to which the Lorentz vectors of all other jets within $\Delta R=\sqrt{(\Delta \eta)^{2}+(\Delta \phi)^{2}}<0.1$ are summed up. For these "wide jets", the conditions $\left|\Delta \eta^{\text {wide }}\right|<1.3$ and $m_{j j}^{\text {wide }}>890 \mathrm{GeV}$ are demanded.

Figure 5 shows the dijet mass distribution normalised to the integrated luminosity of the sample $\left(d \sigma / d m_{j j}\right)$. The prediction of multijet background is normalised to the data with a factor of 1.23. A method based on the fit of data is used to estimate the background from multijet production.

No significant deviations of the data from the background hypothesis are observed. Upper limits are set on the production cross section for different resonance final states (gluon-gluon, quark-gluon, quark-quark) as a function of the resonance mass: $Z_{S S M}^{\prime}$ is excluded below $1.70 \mathrm{TeV}$, $W_{S S M}^{\prime}$ bosons below $2.20 \mathrm{TeV}$, and RS gravitons below $1.60 \mathrm{TeV}$ for $k / \bar{M}_{P l}=0.10$. Figure 5 (right, (a)) shows the exclusion limit plot for the models quoted above plus other models that are discussed in [16]. Figure 5 (right, (b)) also shows $95 \%$ CL lower limits on $M_{t h}\left(M_{Q B H}^{\min }\right.$ in the figure) as a function of the Planck scale $M_{D}$ and the number of extra dimensions $n$ for the QBH model. Lower bounds between $5.00(n=1)$ and $6.36(n=1) \mathrm{TeV}$ are set on the masses of QBH. 

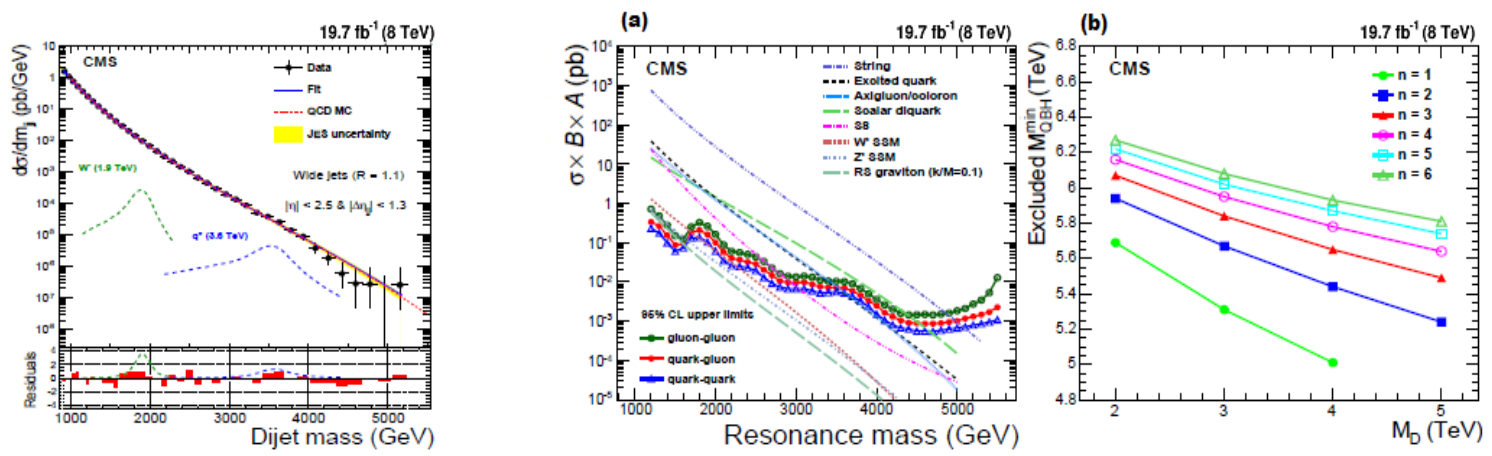

Figure 5: Left: The dijet mass spectrum for data (points) and SM expectations (histograms). Right: The 95\% CL exclusion regions for (a) $Z_{S S M}^{\prime}, W_{S S M}^{\prime}$, RS and other models discussed in [16], (b) QBH model mentioned in Section 3.

\section{Conclusions}

Several measurements from CMS at $\sqrt{s}=8 \mathrm{TeV}$ with $19.7 \mathrm{fb}^{-1}$ have been presented in search for new physics beyond the SM analysing final states with two particles and particles plus $\not_{T}$ in the following channels: $e e$ and $\mu \mu, e \mu, e \mu+\not_{T}, e$ or $\mu$ or $\tau_{h}+Z_{T}$, and $j j$. In all the cases there was a good agreement between the observations and the SM expectations and 95\% CL upper bounds have been set on parameters from a number of new physics models, as summarised in Table 1.

\begin{tabular}{|c|c|c|c|c|c|c|c|}
\hline & $Z^{\prime}$ (Mass) & $W^{\prime}$ (Mass) & $\tilde{v}_{\tau}$ (Mass) & $\operatorname{ADD}\left(M_{S}\right)$ & RS (Mass) & UED (Mass) & $\mathrm{QBH}\left(M_{t h}\right)$ \\
\hline $\begin{array}{l}e e+ \\
\mu \mu\end{array}$ & $\begin{array}{c}2.90(\mathrm{SSM}) \\
2.57(\psi)\end{array}$ & & & $\begin{array}{l}4.90(n=3) \\
3.30(n=7)\end{array}$ & $\begin{array}{l}2.73\left(k / \bar{M}_{P l}=0.10\right) \\
2.35\left(k / \bar{M}_{P l}=0.05\right) \\
1.27\left(k / \bar{M}_{P l}=0.01\right)\end{array}$ & & \\
\hline$e \mu$ & & & $\begin{array}{c}1.28 \\
\left(\lambda_{311}^{\prime}=\lambda_{132}=0.01\right)\end{array}$ & & & & $\begin{array}{l}2.81(n=1) \\
3.63(n=6)\end{array}$ \\
\hline$e \mu+E_{T}$ & $\begin{array}{c}1.30(\mathrm{SSM}) \\
0.81(\psi)\end{array}$ & & & $\begin{array}{l}3.33(n=3) \\
2.23(n=7)\end{array}$ & & & \\
\hline $\begin{array}{c}\ell+\not_{T} \\
(\ell=e, \mu)\end{array}$ & & 3.28 (SSM) & & & & $\begin{array}{c}3.71(\mu=10) \\
1.74(\mu=0.05)\end{array}$ & \\
\hline$\tau_{h}+Z_{T}$ & & 2.70 & & & & & \\
\hline$j j$ & 1.70 (SSM) & $2.20(\mathrm{SSM})$ & & & $1.60\left(k / \bar{M}_{P l}=0.10\right)$ & & $\begin{array}{l}5.00(n=1) \\
6.30(n=6)\end{array}$ \\
\hline
\end{tabular}

Table 1: The 95\% exclusion limits (in $\mathrm{TeV}$ ) for the parameters of the main models considered in the text.

\section{References}

[1] P. Langacker, Rev. Mod. Phys 81 (2009) 1199-1228 [arXiv: 0801.1345$].$

[2] J. Hewett and T. Rizzo, Phys. Rept. 183 (1989) 193.

[3] S.P. Martin, Adv. Ser. Dir. High Energy Phys. 21 (2010) 1-153 [arXiv: hep-ph/9709356].

[4] N. Arkani-Hamed, S. Dimipoulos, and G. Dvali, Phys. Lett. B 429 (1998) 263-272 [arXiv: hep-ph/ 9803315 ].

[5] K. Kong, S. C. Park, and T. G. Rizzo, JHEP 04 (2010) 081 [arXiv: 1002 . 0602].

[6] L. Randall and R. Sundrum, Phys. Rev. Lett. 83 (1999) 3370 [arXiv: hep-ph/ 9905221 ].

[7] E. Eichten, K. Lane, and M. Peskin, Phys. Rev. Lett. 50 (1983) 811.

[8] CMS Collaboration, JINST 3 (2008) S08004.

[9] CMS Collaboration, JHEP 04 (2015) 025 [arXiv: hep-ex/1412 . 6302].

[10] CMS Collaboration, CMS-PAS-EXO-13-002.

[11] D. M. Gingrich, J.Phys. G37 (2010) 105008 [arXiv: 0912 . 0826].

[12] CMS Collaboration, CMS-PAS-EXO-12-046.

[13] CMS Collaboration, Phys. Rev. D 91 (2015) 092005 [arXiv: hep-ex/1408.2745].

[14] CMS Collaboration, CMS-PAS-EXO-12-011.

[15] L. Edelhäuser and A. Knochel, Submitted (2014) [arXiv:hep-ph/1408.0914].

[16] CMS Collaboration, Phys. Rev. D 91 (2015) [arXiv: hep-ex/1501.04198]. 